\title{
Considerazioni a proposito della «giuridicità» delle indulgenze
}

\section{Considerations Regarding the «Juridicity» of Indulgences}

\begin{abstract}
Massimo DEL POZZO
Profesor Extraordinario de Derecho Constitucional Canónico Pontificia Università della Santa Croce. Facoltà di Diritto Canonico. Roma delpozzo@pusc.it
\end{abstract}

Resumen: El artículo pretende comprender la dimensión jurídica de las indulgencias. El análisis parte del origen y de la configuración histórica de la remisión de la pena temporal, para delinear la génesis, la estructura y la colocación en el actual código latino. Se resalta cómo lucrar las indulgencias escapa a la lógica intersubjetiva y obligatoria típica del derecho (pertenece a la esfera moral y espiritual). La juridicidad de las indulgencias no deriva solamente de la configuración y reglamentación eclesial de la concesión, sino también de la institucionalidad de la Iglesia y de la esencia comunitaria y solidaria del bien. Más allá de posibles consideraciones acerca del contenido y de la colocación de la figura de iure condendo, se sugiere un incremento sustancial de la praxis de las indulgencias que subraye, sobre todo, la promoción del perdón sacramental y de la maduración de las necesarias disposiciones, evitando toda interpretación legalista 0 formalista de la disciplina.

Palabras clave: Indulgencias, Cann. 992-996 CIC, Potestad vicaria, Institucionalidad y ministerialidad de la Iglesia.
Abstract: This article seeks to grasp the juridical dimension of indulgences. Its analysis begins with the origin and historical configuration of the remission of temporal punishment, in order to delineate the genesis, structure and positioning of their current provision in the Latin Code. It is notable how the use of indulgences eludes the intersubjective and obligatory logic that is typical of law (in that it regards the moral and spiritual sphere). The juridicity of indulgences, however, is derived not only from the configuration and ecclesial regulation of their concession, but also from the institutionality of the Church and from their community implication, in support of the good. Beyond possible reconsiderations de iure condendo of the content and positioning of the figure, there is the suggestion of a substantial increase in the praxis regarding indulgences in order to underline, above all, the promotion of sacramental forgiveness and the maturation of the necessary dispositions, evading every sort of legalistic or formalistic interpretation of their legislation.

Keywords: Indulgences, Cann. 992-996 CIC, Vicarious Power, Institutionality and Ministeriality of the Church. 


\section{UN'INDAGINE MIRATA E PUNTUALE}

$\mathrm{L}$ a conclusione dell'Anno straordinario della Misericordia ${ }^{1}$, come ogni ricorrenza giubilare e, in parte, a maggior ragione ${ }^{2}$, offre una proficua opportunità per riconsiderare la dimensione giuridica dell'istituto dell'indulgenza. La «remissione di pena temporale» infatti si inserisce esplicitamente nel contenuto e nelle aspirazioni dell'invito al ritorno alla casa del $\mathrm{Pa}$ dre. Il tesoro di grazie che la Chiesa dispensa ai suoi fedeli evidentemente è legato sempre alla fedele amministrazione e generosa profusione del patrimonio salvifico. Il carattere benefico e grazioso del dono o, piuttosto, dell'estinzione del debito di pena non esclude dunque il rilievo autoritativo della facoltà e previsione della concessione ${ }^{3}$. Il punto più stimolante e interessante è però accertare se la giuridicità dipende solo dalla portata sociale delle indulgenze (l'origine e la regolamentazione della fattispecie) o dalla struttura stessa e dall'atteggiarsi del bene considerato ${ }^{4}$. $\mathrm{Al}$ di là la del fondamento e della prerogativa della concessione, la consistenza della realtà e, soprattutto, della relazione indulgenziante sembrerebbe sfuggire alla logica intersoggettiva e obbligatoria tipica del diritto. L'operatività dell'indulgenza non pare riguardare in pratica la sfera del debito in foro esterno ma quella spirituale e morale ${ }^{5}$. L'auspicabile convergenza e accostamento tra devozione del fedele e giustizia non dovrebbe portare a un'indebita sovrapposizione o confusione di piani e prospettive ${ }^{6}$. Interessa quindi esplorare la motivazione e la ratio sistematica delle attuali scelte legislative e i possibili sviluppi de iure condendo.

La risalenza storica e la valenza istituzionale delle indulgenze non bastano a giustificarne l'inserimento nella normativa codiciale, tra l'altro come una sor-

${ }^{1}$ Cfr. Francesco, bolla Misericordiae vultus, 11 aprile 2015, AAS 107 (2015) 399-420.

2 Il richiamo è rafforzato della specialità dell'occasione e dall'accentuazione del senso della convocazione (bolla di indizione del Giubileo straordinario della misericordia).

3 Cfr. J. Hervada, Le radici sacramentali del diritto canonico, Ius Ecclesiae 17 (2005) 635-636.

${ }^{4} \mathrm{Si}$ tratta di accertare se l'aspetto giuridico è intrinseco e connaturato alla dinamica relativa a tale bene della comunione.

${ }^{5}$ Osservava a proposito della nozione F. M. Cappello: «Dicitur a) remissio coram Deo, et non coram sola Ecclesia, v. g. poenae ex sententia iudiciali debitae. Proinde, cum agatur de remissione poenae temporalis in foro Dei, errarunt ii omnes, qui indulgentias acceperunt pro remissione poenae in foro solius Ecclesiae debitae» (Tractatus canonico-moralis de sacramentis, II, Marietti, Taurini-Romae 1953, 660).

${ }^{6}$ Cfr. M. DEL Pozzo, La giustizia nel culto. Profili giuridici della liturgia della Chiesa, Edusc, Roma 2013, 25-27. 
ta di appendice della disciplina sacramentale ${ }^{7}$. Una mera esigenza dottrinale e catechetica o l'ossequio alla tradizione canonica meritano comprensione e apprezzamento ma costituiscono un argomento debole e poco convincente. Il ridimensionamento o l'espunzione della relativa normativa dalle codificazioni vigenti ${ }^{8}$, con la discussione che ne è alla base, come tra poco vedremo, ben evidenzia l'incertezza e problematicità della consistenza giuridica della figura. Con maggior incisività o radicalità vale la pena allora di chiedersi perché il Codificatore latino debba riservare spazio e attenzione (un apposito titolo) all'economia «parasacramentale» indulgenziale. Alla radice c'è probabilmente un insoluto chiarimento circa la questione epistemologica e l'essenza della disciplina e della doverosità nell'agire del cristiano ${ }^{9}$. La preoccupante «diserzione» dei moralisti dalla sacramentaria in senso lato ha prodotto un vuoto di scienza e un'assenza di specifici approfondimenti. I residui della matrice autoritaristica e imperativa dell'ordine sociale giusto del popolo di Dio probabilmente non aiutano la percezione dell'autonoma stringenza dell'Enchiridion Indulgentiarum e l'incentivo o il recupero dell'antica pratica della penitenza riparatrice.

Il senso dell'indagine non è ovviamente quello di inficiare o minare il ricorso alle indulgenze ma anzi di promuovere e incrementare l'esperienza e l'esercizio del perdono ecclesiale, evitando appunto malintese interpretazioni o restrizioni «giuridiciste». Solo l'adeguata collocazione del fattore giuridico aiuta d'altronde a cogliere la ricchezza e bontà della dimensione di giustizia nella vita di fede ${ }^{10}$. L'indubbio presupposto giuridico o, piuttosto, istituziona-

${ }^{7}$ L'abituale collocazione manualistica o commentaristica segue l'assetto codiciale, considera le indulgenze un complemento del sacramento della Penitenza. Ci sembra più confacente invece la scelta di B. F. Pighin di inserire il tema tra gli atti di culto diversi dai sacramenti (Diritto sacramentale, Marcianum Press, Venezia 2006, 359-367 [Cap. II. Le indulgenze, le preghiere di guarigione e l'esorcismo]).

8 «Mentre la disciplina anteriore trattava la materia sull'indulgenza in 26 canoni (CIC, cann. 911936), il Codice vigente lo fa in soli 6 canoni (cann. 992-997); quest'ultimi riportano inoltre, quasi letteralmente, i documenti menzionati sopra. il Codice Orientale non tratta affatto la materia delle indulgenze» (E. Frank, I Sacramenti dell'Iniziazione, della Penitenza e dell'Unzione degli infermi. Commento ai Canoni 834-1007 del Codice di Diritto Canonico, Urbaniana University Press, Città del Vaticano 2012, 182).

${ }^{9}$ Cfr. M. DEL Pozzo, La doverosità liturgica, morale e giuridica del culto ecclesiale, Ius Ecclesiae 21 (2009) 549-568; C. J. ERRázURIZ M., Lintrinseca doverosità liturgica e giuridica del culto ecclesiale, in Diritto e norma nella liturgia, E. BAURA - M. DEL POZZo (a cura di), Giuffrè Editore, Milano 2016, 29-58.

10 Il problema, come più volte rilevato, non è il «troppo» diritto, ma, per così dire, il «cattivo» diritto (l'erronea o equivoca percezione del fattore giuridico), cfr. ad es. M. DEL Pozzo, La dimensione giuridica della liturgia. Saggi su ciò che è giusto nella celebrazione del mistero pasquale, Giuffrè Editore, Milano 2008, 110-111 (spec. nt. 8). 
le della concessione non deve far perdere di vista lo spirito e il carattere prettamente penitenziale ed espiatorio della pratica e il suo valore individuale e interiore ${ }^{11}$. Ci sono parecchi testi sulla concezione e disciplina storica e attuale delle indulgenze ${ }^{12}$, ma, ci sembra, pochi studi di impronta essenziale o fondamentale sull'istituto. La necessità della previsione normativa basilare si dà normalmente per scontata e assodata, mentre proprio la logica codificatoria dovrebbe indurre a un maggior rigore e precisione concettuali e definitori. Il presente contributo vorrebbe perciò colmare o integrare l'orizzonte di senso e di significato nella percezione degli estremi della figura sub specie iuris.

\section{ORIGINE E CONFIGURAZIONE STORICA DELL'ISTITUTO}

La concessione delle indulgenze appartiene allo sviluppo organico dell'economia salvifica o alla progressiva acquisizione della ricchezza e virtualità del patrimonio di grazia. L'approfondimento della dottrina teologica sugli effetti del peccato (distinzione tra debito di colpa e di pena) si coniuga con la positiva ricerca di purificazione e riscatto per sé e per i defunti e l'assicurazione della liberazione dalla punizione temporale ${ }^{13}$. Le crociate e, più in generale, i pellegrinaggi sono stati probabilmente il maggior fattore di stimolo all'impegno riabilitativo ed espiatorio in un momento i cui il richiamo alla santità coincideva soprattutto con la coerenza esterna e la percepibilità dell'appartenenza alla comunità cristiana. La concessione della remissio nasce

${ }^{11}$ L'indulgenza non è un espediente o un'agevolazione giuridicista dell'economia salvifica, ma una conseguenza abbastanza diretta dell'autenticità e operosità della compunzione mediata dal giudizio e dal supporto della Chiesa.

12 Cfr. ad es. C. CACCIARI, Le indulgenze, Edizioni Sabinae, Cantalupo in Sabina (RI) 2009 (precedente edizione della stessa tesi: Odierno sistema giuridico delle indulgenze e proposte di interpretazione, Roma 2007); G. DE PASCAL, Les indulgences. Doctrine et histoire, Bloud et C., Paris 1908; A. H. M. LÉPICIER, Le indulgenze. Loro origine, natura, e svolgimento, Direzione della Biblioteca del clero, Siena 1897; B. Poschmann, Der Ablass im Licht der Bussgeschichte, Bonn 1948 (Theophaneia 4); E. F. Regatillo, Las indulgencias, Sal Terrae, Santander 1947; R. D. Ruiz MaINardi, La disciplina de las indulgencias en el siglo XX, Pontificia Universitas Sanctae Crucis, Romae 2000; W. TAUWINKL, La discussione teologica sulle indulgenze dal Concilio Vaticano II fino ad oggi, Galaxia Gutenberg, Târgu Lapus 2005. Si prescinde in questa sede dall'approfondimento delle questioni teologiche connesse.

${ }^{13}$ La storia manifesta che le indulgenze non sono tanto una spinta o un incentivo dell'autorità quanto una sentita esigenza di conversione e di fattiva riabilitazione del popolo cristiano. Non è casuale che la «scoperta» del rimedio remissivo si inserisca nella progressiva enucleazione e affinamento del cammino penitenziale. La fruizione delle indulgentiae appare in questa linea come un corollario o un'esplicitazione (e non una scorciatoia o un surrogato) dell'approfondimento del contenuto e dell'estensione della misericordia divina. 
quindi dalla vita di fede e dalla pietà popolare più che dalla decisione dell'autorità e dalla ufficialità del riconoscimento. Il regime delle indulgenze comunque prende piede e si sviluppa proprio nell'ottica legalistica e gerarchica del sistema e della mentalità medievale ${ }^{14}$. La crisi protestante, com'è noto, evidenzia subito e in maniera aggressiva i limiti e le deviazioni pratiche del modello questorio ${ }^{15}$. Il Concilio di Trento peraltro, pur riconoscendo e correggendo gli abusi, afferma perentoriamente la fondatezza e validità della dottrina cattolica ormai affermata e consolidata ${ }^{16}$. Nella modernità l'istituto conosce perciò un affinamento e un'attenta regolamentazione fino al riordino attuato dal beato Paolo VI ${ }^{17}$. Non si può evidentemente indagare la natura e la disciplina delle indulgenze prescindendo completamente dal dogma e dalla storia ${ }^{18}$. In questa sede non abbiamo la pretesa di ricostruire compiutamente il cammino percorso, vogliamo comunque proporre almeno un sommario accenno alle tappe di configurazione, consolidazione, fissazione e dif-

14 «Certo è che vere indulgenze, concesse per elemosine o per visite di devozione a chiese e altari..., cominciano - ma sono ancora poche - nel secolo XI, e si moltiplicano nel secolo XII e dopo» (E. DAL COVOLO, Per una storia dell'indulgenza. Nota in margine alla Bolla di indizione del Grande Giubileo, Salesianum 61 [1999] 823). Lo studio storico più documentato e accreditato sull'istituto resta comunque N. PAulus, Geschichte des Ablasses im Mittelalter, I-III, Paderborn 1922-1923, rist. WBG, Darmstadt 2000. Occorre considerare che lo spiccato legalismo e autoritarismo che circonda il fenomeno non indica comunque una sorta di inautenticità o imposizione nell'estensione e diffusione del costume.

${ }^{15}$ I quaestores erano deputati alla proclamazione e raccolta delle offerte indulgenzianti, talora venalmente e in modo poco avveduto, cfr. A. MigLiavacCA, Le indulgenze, Quaderni di diritto ecclesiale 11 (1998) 161-162.

16 «Il potere di conferire le indulgenze è stato accordato dal Cristo alla chiesa che, fin dai tempi più antichi, ha usato di questa facoltà a lei divinamente concessa (cfr. Mt 16,19;18,18). Per questo il santo sinodo insegna e comanda di mantenere nella chiesa quest'uso, molto salutare per il popolo cristiano e approvato dall'autorità dei sacri concili, e colpisce con l'anatema quelli che definiscono inutili le indulgenze o negano alla chiesa il potere di concederle. Tuttavia, desidera che nel concedere le indulgenze si usi moderazione... per evitare che la troppa facilità nel concederle indebolisca la disciplina ecclesiastica. Desiderando poi emendare e correggere gli abusi che vi si annidano e in forza dei quali la bella parola indulgenza viene bestemmiata dagli eretici, col presente decreto stabilisce, in generale, la completa abolizione di tutti gli indegni traffici di soldi fatti per ottenerle» (CONCILIO DI TRENTO, Decreto sulle indulgenze, 4 dicembre 1563, in Dz-Sch. 1835). Il IV Concilio Lateranense (1215) già aveva cercato di correggere abusi e cedimenti operativi (cfr. W. H. Woestman, Sacraments. Initiation, Penance, Anointing of the Sick. Commentary on Canons 840-1007, Saint Paul University, Ottawa 2004, 297-298).

${ }^{17}$ Cfr. PAOLO VI, cost. ap. Indulgentiarum doctrina, 1 gennaio 1967, AAS 59 (1967) 5-24 [= ID].

${ }_{18}$ Per un inquadramento storico dell'istituto, oltre a N. Paulus, Geschichte des Ablasses..., cfr. anche P. Galtier, De paenitentia. Tractatus dogmatico-bistoricus, apud aedes Universitatis Gregorianae, Romae 1956, 518-546; B. Poschmann - F. Courtney, Penance and the anointing of the sick, Herder-Burns and Oates, Freiburg im Breisgau-London 1964, 210-232; J. A. JungManN, Die lateinischen Bussriten in ibrer geschichtlichen Entwicklung, F. Rauch, Innsbruck 1932. 
fusione delle indulgenze che funga da premessa e sfondo dell'attuale ricezione e sistemazione normativa.

Fino al secondo millennio cristiano la disciplina indulgenziale non giunge ancora a configurarsi in senso proprio e definito ${ }^{19}$. La remissione della pena appare infatti inscindibilmente collegata all'assoluzione sacramentale. La straordinarietà e il rigore della penitenza, com'è noto, rendono sporadico o diradano il ricorso alla Riconciliazione ma avvalorano contestualmente l'esigenza dell'espiazione e della reintegrazione. Le incertezze nella ricostruzione storica non permettono di determinare esattamente la prassi e i contenuti del regime penitenziale ${ }^{20}$, il perdono si associa tuttavia all'esplicitazione del serio desiderio di conversione e all'adempimento previo delle opere penitenziali (la remissione della pena temporale è quindi compresa, almeno in parte, nel percorso di contrizione e riparazione). Sin dai primi secoli ad ogni modo la potestà episcopale e l'appello all'intercessione dei martiri mirano a facilitare e incentivare la confessione delle colpe gravi e la riconciliazione ecclesiale ${ }^{21}$. La pastoralità della cura d'anime si esprime anche nella relaxatio e commutatio della penitenza prevista ${ }^{22}$. La preghiera, il digiuno e, soprattutto, l'elemosina o l'esercizio della misericordia e della pietà contribuiscono ad attenuare o stemperare la durezza delle punizioni. Le c.d. redemptiones e peregrinationes romanae costituiscono perciò i prodromi o antesignani del regime concessorio successivo. Anche la frequenza delle absolutiones (l'inserimento di formule espiatorie) manifesta la forte sensibilità per la riparazione. Tali figure sono però propedeutiche o finalizzate al perdono sacramentale. Nel primo millennio dunque non appare ancora la «relativa» autonomia dell'indulgentia, la garanzia ex

19 «Las indulgencias bajo esta forma definitiva, comenzaron a concederse en el siglo XI y, con ocasión de diversas obras: la dedicación de un templo, la construcción de un puente, la defensa del pueblo cristiano, la custodia de la paz, los sacrificios, las oraciones, los pergrinajes, etc.; aplicables en algunos casos, a los fieles difuntos» (R. D. RUIZ MAINARDI, Indulgencias, in DGDC IV, 551). Dal Covolo a proposito degli istituti precedenti (riconciliazione, mitigazione, diminuzione e commutazione della penitenza sacramentale) parla di «prodromi dell'indulgenza» (Per una storia..., cit., 821-823).

${ }^{20}$ Cfr. A. GiacobBi, Storia della disciplina penitenziale antica, Roma 1976; R. LoRÌA, La Penitenza nei secoli. Excursus storico sull'istituto penitenziale nella Chiesa, in L. LIGIER ET AL. (a cura di), La penitenza. Dottrina, storia, catechesi e pastorale, Elledici, Torino 1967, 177-224; C. VOGEL, Il peccatore e la penitenza nella Chiesa antica, Elledici, Torino 1967; IDEM, Il peccatore e la penitenza nel medioevo, Elledici, Torino 1988.

${ }^{21}$ Cfr. anche C. CACCIARI, Le indulgenze, 11-15.

${ }^{22}$ Cfr. E. Dal Covolo, Per una storia..., cit., 821-823. Il più efficace incentivo della pratica penitenziale è dovuta all'introduzione della penitenza tariffata. L'uso dei penitenziali attesta peraltro la ripresa e la diffusione del perdono sacramentale nel costume del popolo cristiano. 
auctoritate e la prerogativa dell'azzeramento (anche totale) del debito di pena; i suffragi inoltre esauriscono le forme di intercessione a favore dei defunti.

Nel basso medioevo si assiste alla formalizzazione e al fiorire delle indulgenze $^{23}$. Le crociate offrono probabilmente l'occasione più propizia per la riabilitazione dei peccatori e la massima espansione possibile di elargizioni di grazia $^{24}$. La pratica si estende contestualmente ai pellegrinaggi e, in seguito, al finanziamento delle opere pubbliche, sociali e assistenziali. Col tempo l'esborso pio o caritatevole diviene il prototipo forse più rappresentativo di opera indulgenziata, ciò comporta il rischio non troppo recondito di mettere in ombra il primato della grazia e il richiamo alla contrizione (la maggior diffusione e la mitigazione aveva reso pure meno ostica e sentita il ricorso alla penitenza). Il popolo cristiano con sincera pietà cerca e lucra le indulgenze, in origine però come una vera necessità spirituale poi, sempre più, come un costume sociale meritevole. I Papi normalmente assecondano la necessitas o l'utilitas Ecclesiae con speciali concessioni e assicurazioni. La teologia scolastica approfondisce la dottrina sugli effetti del peccato, sulla sovrabbondanza dei meriti della Redenzione, sulla comunione dei santi, sulla potestà delle chiavi, ecc. che fornisce la sicura base e fondamento delle indulgenze. Il magistero in materia si consolida con il IV Concilio di Lateranense (1225) e poi con quelli di Lione e di Vienne (1245, 1274, 1311-1312). La maternità della Chiesa apre le porte all'indulgenza plenaria in articulo mortis prima per i crociati e poi per tutti. L'applicazione delle indulgenze per i defunti si diffuse rapidamente nel popolo fedele, senza un'espressa approvazione ecclesiastica. Solo alla fine del $\mathrm{XV}$ sec. si concede esplicitamente l'indulgentia in modum suffragii, supportata dalla necessaria speculazione teologica ${ }^{25}$.

Le indulgenze risultano pertanto ben esplorate nella dottrina, misurate nella regolamentazione ed estese nella pratica sin dal basso medioevo. Gli abusi operativi e le deviazioni concettuali hanno finito però per introdurre, attraverso delle questue disavvedute e dei questori impreparati, l'impressione di

${ }^{23}$ Cfr. P. Galtier, Les indulgens: origine et nature, à propos d'un ouvrage récent, Gregorianum 31 (1950) 258-274.

24 «Quicumque pro sola devotione, non pro honoris vel pecuniae adeptione, ad liberandam Ecclesiam Dei, Jerusalem profectus fuerit, iter illud pro omni poenitentia reputetur» (c. 2, Concilio di Clermont [1095], in J. D. MANsi (a cura di), Sacrorum Conciliorum nova et amplissima collectio, XX, Akademische Druck- und Verlaganstalt Graz, Graz 1960, col. 816).

25 Cfr. anche J. GoÑ GaZTAMbide, Las primeras indulgencias de difuntos, Antologica Annua 2 (1954) 378-391. 
una specie di «perdono tariffato» o di un «mercato della grazia» che disdice all'autentico senso della remissione penitenziale. Il Concilio di Trento non ha fatto altro che ribadire la validità e il valore dell'insegnamento tradizionale, correggere gli errori rilevati e indicare misure disciplinari ${ }^{26}$. La pratica delle indulgenze di fatto non ha sortito seri contraccolpi fino alla post-modernità. La crescente devozione popolare (non sempre proporzionale all'incremento della pietà ${ }^{27}$ ha anzi moltiplicato il numero e la facoltà di guadagnare indulgenze con taluni eccessi casuistici e prescrittivi (la determinazione quantitativa delle indulgenze parziali). Le direttive di Trento portarono anche alla creazione della $S$. Congregatio pro indulgentiis et reliquiis. Il Dicastero fu poi accorpato alla Congregazione dei Riti da S. Pio X, l'attribuzione passò quindi al Santo Uffizio e infine alla Penitenzieria Apostolica (fermo restando la persistente competenza del Sant'Offizio nel profilo dottrinale) ${ }^{28}$. La previsione di uno specifico organismo curiale è un eloquente dimostrazione dell'interesse e della viva preoccupazione ecclesiale per la materia. La codificazione piano benedettina, dal canto suo, ha sintetizzato ed esposto tutta la dottrina e disciplina generale sulle indulgenze nei 37 canoni del De indulgentiis ${ }^{29}$.

\section{L'ATTUALE PREVISIONE CODICIALE:} GENESI, 'RATIO', STRUTTURA E COLLOCAZIONE

Il Concilio Vaticano II non si è interessato specificamente del tema delle indulgenze ${ }^{30}$. Taluni Padri comunque manifestarono il desiderio di un approfondimento teologico e di una profonda revisione disciplinare dell'isti-

${ }^{26}$ Cfr. supra nt. 16. Oltre alla soppressione dei quaestores si riservò la facoltà di concedere indulgenze solo agli Ordinari (CONCILIO DI TRENTO, XXI sess., 16 luglio 1562, Decretum de reformatione, c. 9, in Conciliorum Oecumenicorum Decreta, Edizioni Dehoniane, Bologna 1991, 731-732).

27 «La pietà dei fedeli stimava più alto il valore delle indulgenze, quando esse erano ancora molto rare. D'altra parte, la pietà medievale si esplicò in una quantità innumerevole di devozioni particolari, di confraternite e associazioni, e tutto ciò diede occasione alla richiesta di sempre nuove indulgenze» (E. DAL Covolo, Per una storia..., cit., 825).

${ }^{28}$ Con Breve del 6 luglio 1669 Clemente IX costituì la S. Congregazione per le Indulgenze e le Reliquie; Pio X unì tale Congregazione alla Congregazione dei Riti (28 gennaio 1904); la materia passo quindi al Sant'Uffizio con l'Ordinamento della Curia Romana (1908); Benedetto XV trasferì la Sezione delle Indulgenze dal Sant'Uffizio alla Penitenzieria Apostolica (25 marzo 1915), cfr. C. CACCIARI, Le indulgenze, 25-31.

29 Cfr. CIC 17, Liber tertius. De rebus, Pars prima. De Sacramentis, Titulus IV. De poenitentia, Caput V. De indulgentiis (cann. 911-936).

30 È significativo il fatto che la cost. Sacrosanctum Concilium, che pure ha esaminato a tutto tondo lo spettro della realtà cultuale, non menzioni mai le indulgenze. 
tuto che ripristinasse il senso della remissione e del perdono nell'epoca attuale ed evitasse persistenti incomprensioni ed equivoci ${ }^{31}$. Le decisioni e gli interventi operativi furono però demandati alla valutazione e alla ponderazione della Sede Apostolica. La consultazione delle diverse Conferenze episcopali svolta dalla Penitenzieria Apostolica offrì inoltre spunti e indicazioni che trovarono un tempestivo e attento riscontro nelle risoluzioni del beato Paolo VI. La cost. ap. Indulgentiarum doctrina del $1^{\circ}$ gennaio 1967 consolida e ravviva appunto l'insegnamento tradizionale della Chiesa e stabilisce i nuovi criteri del regime indulgenziale alla luce delle acquisizioni liturgiche, sacramentali ed ecclesiologiche maturate nell'assise ecumenica ${ }^{32}$. Le statuizioni riguardano soprattutto l'integrazione delle disposizioni richieste, l'incentivo al fervore e alla preparazione, la precisazione del contenuto delle indulgenze parziali (l'eliminazione della determinazione dei giorni e degli anni), la riduzione delle indulgenze plenarie, la soppressione delle indulgenze c.d. reali e locali ${ }^{33}$. Il Pontefice comunque non dispone un cambiamento radicale del sistema legale vigente ${ }^{34}$. Alla norma quadro di riferimento fa seguito la promulgazione del nuovo Enchiridion indulgentiarum del 29 giugno $1968^{35}$.

La formulazione codiciale riflette il cambiamento di prospettiva intervenuto ma manifesta pure incertezze e ripensamenti circa $i$ contenuti e lo sviluppo del testo. Spicca subito la mutata impostazione concettuale rispetto all'analiticità e minuziosità del CIC $1917^{36}$. Nel processo redazionale si registra comunque un passaggio abbastanza brusco tra le prime versioni e lo schema del 1980 e quelli

${ }^{31}$ Cfr. A. Migliavacca, Le indulgenze, 163; W. TauWInKL, La discussione teologica sulle indulgenze..., cit., 95-111. A. CALvo ESPIGA, Algunas orientaciones actuales de la teología de las indulgencias, Burgense 21 (1980) 417-449, illustra la profonda influenza della dottrina di K. Rahner sul tema.

32 La costituzione apostolica si articola in 12 punti di carattere speculativo e magisteriale che ribadiscono e attualizzano il valore delle indulgenze e in 20 norme direttive che guidano la revisione dell'Enchiridion indulgentiarum.

33 «Nel redigere le nuove norme si è cercato in particolar modo di stabilire una nuova misura con l'indulgenza parziale, di apportare una congrua riduzione al numero delle indulgenze plenarie e di dare alle indulgenze cosiddette reali e locali una forma più semplice e più dignitosa» (ID 11).

34 «Le disposizioni del codice di diritto canonico e dei decreti della santa sede riguardanti le indulgenze, in quanto sono conformi alle nuove norme, restano invariate» (ID 11).

35 Paenitentiaria Apostolica, Enchiridion indulgentiarum. Normae et concessiones, Typis Polyglottis Vaticanis, Città del Vaticano 1968.

36 Il mutamento non è solo quantitativo ma soprattutto stilistico e qualitativo nel tenore delle prescrizioni. Nel CIC 1917 il titolo De indulgentiis fissava e compendiava la disciplina generale dell'istituto, nel CIC 1983, al di là della revisione normativa già intervenuta, riepiloga ed estrapola solo i tratti salienti della figura. 
successivi ${ }^{37}$. Il desiderio di semplificare e snellire il disposto originariamente avevano portato solo a contenere la regolamentazione in un più modesto numero di canoni. La linea di lavoro inizialmente seguita era semplicemente la trasposizione e adeguazione delle normae della cost. ap. Indulgentiarum doctrina. Nella sessione del marzo del 1972 si approntò uno schema iniziale di 24 canoni (cann. 155-179) ${ }^{38}$, che a seguito di alcune soppressioni e accorpamenti, configurò i 18 canoni (cann. 162-180) dello schema de sacramentis approvato $^{39}$. A seguito di un'incisiva rivisitazione dei criteri di elaborazione da parte delle Plenaria il testo venne però ridotto all'essenziale. L'operazione di revisione destò un po' di contrarietà e rammarico nei Consultori ${ }^{40}$. Una logica di coerenza e omogeneità impose tra l'altro un'ulteriore diminuzione di canoni, giungendo così - con l'aggiunta della norma di rinvio conclusiva (l'attuale can. 997) - ai sei poi definitivamente promulgati. Un deciso «salto in avanti» pertanto è maturato in corso d'opera e ha superato l'intenzione iniziale dei redattori.

Lo svolgimento dei lavori manifesta un moderato intento di ripensamento e superamento dell'approccio precedente. Più interessante è però la questione concettuale legata alla natura e struttura della previsione normativa. Nella prima Disceptatio de indulgentiis vengono affrontate infatti le tre questioni fondamentali esposte nel voto del terzo Consultore: collocazione, ampiezza e testo di base del disposto. Il Consultore esprime riserve circa la sistemazione tradizionale (dopo il sacramento della penitenza) ${ }^{41}$; si pone il dubbio sulla convenienza di una trattazione estesa o sintetica ${ }^{42}$; propone come base di lavoro l'Enchiridion indulgentiarum del 1968 anziché il CIC 1917. La discus-

37 Cfr. E. N. PeTERs, Incrementa in progressu 1983 Codicis iuris canonici, Wilson \& Lafleur, Montréal 2005, 897-904; R. Althaus, Komentar cc. 992-997, in Münsterischer Kommentar zum Codex iuris canonici, Ed. K. Lüdicke, 4, Ludgerus, Essen Januar 2008/Februar 2009 (Geneses des Canons).

${ }^{38}$ Cfr. Communicationes 31 (1999) 321-332.

${ }^{39}$ Cfr. Communicationes 32 (2000) 120.

40 «rendiamo atto, pur con un certo rammarico, che il testo è stato ridotto al minimo, rendendo necessario il ricorso all'Enchiridin indulgentiarum anche per le Norme [...] II. Adottata la regola di ridurre i canoni al minimo, conviene forse omettere anche i due ultimi canoni...» (Communicationes 13 [1981] 439-440).

$41 \ll$ Meo umili iudicio disceptandum esset utrum ordinatio Codicis retinenda sit, vel potius hanc materiam post expletam tractationem de septem sacramentis inserere expediat, quod forsitan melius esset ad confusiones vitandas» (Communicationes 31 [1999] 321).

42 «Quaeritur ergo utrum expediat in novo Codice de hac re longius agere, vel tantum summa capita recolere. Si vero tractationem praefertur pro textu Codicis, tunc documenta recentiora essent in "appendicibus" inserenda, qui agendi modus non videtur "practicus"» (Communicationes 31 [1999] 321). 
sione del marzo del 1972 prosegue con la determinazione delle norme da includere e degli adattamenti da apportare. Il lavoro nella Disceptatio de schemate canonum «de indulgentiis» dell'ottobre 1972 si concentra ancora sull'affinamento del testo. Manca un esame approfondito previo dei presupposti e della consistenza della normativa. Qualche rilievo in merito emerge però a proposito del contenuto e del modo di procedere nella redazione ${ }^{43}$. Limpianto «restrittivo» della codificazione emerge anche nel corso dei lavori ${ }^{4}$. Come già accennato, i redattori si attennero anche (con un po' di sofferenza ma in maniera collaborativa) all'indicazione ricevuta di ridurre i canoni al minimo. La più stimolante «provocazione» si desume comunque da un'osservazione alla Relatio conclusiva: «Quidam Pater proponit ut hi canones ad duos tantum reducatur [...] Rationes: a) quia ceteri canones huius Capitis non habent characterem proprie iuridicum; ... $\gg^{45}$. La proposta fu giudicata però troppo drastica ed ecclesialmente inopportuna ${ }^{46}$. Non si ritenne pertanto di procedere a un cambiamento radicale e incisivo dell'assetto ma al semplice ridimensionamento e sfrondamento della normativa.

L'attuale formalizzazione ha quindi un carattere molto essenziale e lineare, conserva tuttavia l'idealità di fornire un sommario riferimento dottrinale più che limitarsi al profilo giuridico dell'istituto. La normativa di conseguenza assume un valore prevalentemente teologico e didascalico. Il disposto dei cann. 992-997 presenta: la definizione d'indulgenza, i tipi d'indulgenza (parziale o plenaria), la destinazione del beneficio (per sé o per i defunti), l'autorità competente a elargirla, la capacità e il modo per lucrare l'indulgenza, il

43 «Rev.mus Secretarius ad. legit normam 1 et declarat quod non est assumendus in Codicem, quia est definitio quae non requiritur in Codicem» (Communicationes 31 [1999] 326). Dopo un ampia discussione nella Sessione IX si conclude però: «Concordant omnes ut definitio de indulgentiis detur in Codicem» (327).

44 A proposito della disceptatio sul can. $159,2^{\circ}$ del primo schema osserva un Consultore: «Consequenter, utile est ut norma 10 maneat in Enchiridion Indulgentiarum, ut exponatur in doctrina de indulgentiis, sed nullo modo necesse est ut in Codice, ubi haec materia sat fuse exponitur, ideoque potius restringi debet quoad extensionem, explicite ponatur» (Communicationes 32 [2000] 56). Conseguentemente: «Rev.mus Secretarius ad proponit ut $2^{\circ}$ supprimatur. Placet omnibus» (ibid.).

45 Communicationes 15 (1983) 212-213. La proposta dei due canoni prevedeva: «Indulgentia, quam Ecclesia quoad poenam temporalem concedit pro peccatis, ad culpam quod attinet, iam deletis, a christifidelibus acquiri potest condicionibus in lege peculiaris statutis»; «Praeter supremam Ecclesiae auctoritatem ii tantum possunt indulgentias elargiri, quibus haec potestas iure agnoscitur aut a Romano Pontifice concessa fuerit» (ibid.).

$46 \ll$ R. Propositio sustineri nequit; reductio nimis drastica esset et materia indulgentiarum dispareret. Textus concordatus est cum S. Paenitentiaria. Nec vitari potest ut Enchyridion Indulgentiarum citetur» (Communicationes 15 [1983] 213). 
rinvio alla specifica legislazione. La logica delle previsioni pare dettata insomma dalla continuità storica e dalla completezza del sistema. La sistemazione ricalca poi quella tradizionale e il necessario collegamento con l'economia penitenziale. La disciplina conserva quindi il ruolo di una sorta di appendice della Riconciliazione. All'emanazione del Codice ha fatto seguito la revisione dell'Enchiridion indulgentiarum nel 1986 e successivamente e nel $1999^{47}$. Anche il Catechismo della Chiesa Cattolica riporta i principi della fede sulle indulgenze, logicamente con un'accentuazione maggiore dei presupposti dogmatici (le pene del peccato, la comunione dei santi e la mediazione della Chiesa) ${ }^{48}$.

\section{LA PROBLEMATICA INTERSOGGETTIVITÀ DELLA FRUIZIONE INDULGENZIALE}

Non vi è dubbio che la giustificazione dell'istituto indulgenziale risieda in un fenomeno potestativo (il potere delle chiavi) e che le relative prescrizioni abbiano un carattere propriamente normativo. Le riserve circa l'inserimento del Caput IV. De indulgentiis nel Codice però non nascono tanto dall'inutilità di duplicare o riportare sommariamente la regolamentazione positiva altrove sviluppata quanto dalla natura stessa della fattispecie considerata. La previsione disciplinare codiciale può far sorgere l'equivoco che la cancellazione della pena e la reintegrazione del peccatore operoso sia un fatto essenzialmente giuridico o segua una logica rivendicativa. Secondo l'ermeneutica dell'ipsa res iusta tommasiana ${ }^{49}$ conviene invece esplorare la giuridicità nella realtà delle indulgenze prima che nella norma positiva. Orbene la dimensione di giustizia dell'indulgenza può essere considerata, per così dire, «radicale o originaria» (è legata all'istituzionalità e mediazione salvifica della Chiesa) ma non pare «essenziale», non stabilisce cioè una relazione di debito. Il beneficio indulgenziale in quanto tale non è né esterno, né intersoggettivo, né doveroso. La definizione stessa d'indulgenza («remissio coram Deo poenae tempo-

47 Cfr. J.-M. GervaIs, La quarta edizione dell'Enchiridion Indulgentiarum, Ius Ecclesiae 12 (2000) 173-187. Le norme dell'Enchiridion sono scese dalle 36 delle 2 edizioni del 1968, alle 29 del 1986, fino alle 26 attuali.

${ }^{48}$ Cfr. Catechismo della Chiesa Cattolica, nn. 1471-1479. I profili indicati corrispondo sostanzialmente alle intitolazioni dei sottoparagrafi. Il Catechismo ribadisce pregiudizialmente il fondamento dell'inquadramento sistematico classico: «La dottrina e la pratica delle indulgenze nella Chiesa sono strettamente legate agli effetti del sacramento della Penitenza» (n. 1471).

${ }^{49}$ Cfr. S. Th., II-II, q. 57, a. 1 , ad 2. 
ralis pro peccatis... ope Ecclesiae» ${ }^{50}$ ) esclude patentemente gli estremi di ogni vincolo obbligatorio interpersonale. Non c'è nulla di più intimo e spirituale della disposizione richiesta per lucrare l'indulgenza plenaria o parziale ${ }^{51}$. L'indulgenza è solo una straordinaria fruizione del tesoro dei meriti di Cristo e dei Santi da parte del fedele riconciliato. L'intervento della Chiesa non configura una specifica prestazione di servizio (a prescindere dall'eventuale rilievo sociale connesso all'opera indulgenziata ${ }^{52}$ ), ma concede una speciale efficacia sanante all'azione del fedele. Il dinamismo del diritto esigerebbe un dovere coram hominibus che, nel caso di specie, è evidentemente inconfigurabile. L'estensione del perdono divino resta un fatto riservato ed eminentemente personale. Il riferimento fondamentale del figura è dunque alla virtù della religione e non all'ordine della giustizia.

La natura e la ratio dell'indulgenza appena delineata si coglie meglio evidenziando lo stacco rispetto all'economia sacramentale. La dottrina sottolinea spesso l'approssimazione o il collegamento dell'istituto con la disciplina dei sacramenti ${ }^{53}$ andrebbe però sottolineata maggiormente la specificità e peculiarità del fenomeno indulgenziale. Per quanto l'organismo sacramentale superi enormemente la prospettiva giuridica ${ }^{54}$, si conforma comunque alla logica dell'esigenza intersoggettiva. La giuridicità dei sacramenti è molto limitativa e parziale (coglie solo un aspetto di un fenomeno misterico e soprannaturale), eppure descrive una dimensione importante dei relativi beni

${ }^{50}$ Can. 992 CIC; cfr. anche Paenitentiaria Apostolica, Enchiridion indulgentiarum. Normae et concessiones, Libreria Editrice Vaticana, Città del Vaticano 1999, n. 1.

51 Si richiede un cuore contrito e pienamente orientato all'amore di Dio.

52 Se ad esempio l'indulgenza è connessa alla visita ad una chiesa, l'impegno ecclesiale, a parte ciò che riguarda la preparazione catechetica e l'aspetto prettamente sacramentale, si sostanzia solo nel favorire la possibilità dell'accesso e nel prevedere un orario congruo di apertura del tempio (cfr. ad es. cann. 1214, 1230, 1234).

53 «A. Connessione di questo trattato con quello della penitenza. La connessione dei due trattati è segnata da due ragioni: - una storica: sempre sono stati trattati insieme. - una dottrinale: - in ragione dell'autorità: è lo stesso potere delle chiavi. - in ragione dell'effetto: vengono rimessi i peccati: - quanto alla colpa: nel sacramento della penitenza. - quanto alla pena temporale: nelle indulgenze» (A. URRU, La funzione di santificare della Chiesa [I Sacramenti], Pontificia Università di San Tommaso d'Aquino in Roma, Roma 1987, 221). Nella stessa linea cfr. anche F. M. CAPPELlo, Tractatus canonico-moralis..., cit., 659; V. DE PAOLIS, Il sacramento della Penitenza, in A. Longhitano - A. Montan - J. Manzanares - V. DE PaOlis - G. Ghirlanda, I sacramenti della Chiesa, Edizioni Dehoniane, Bologna 1989, 233-234; J. A. Marques, Comentario c. 992, in Á. Marzoa - J. Miras - R. Rodríguez-OCaña (a cura di), Comentario exegético al Código de Derecho Canónico, III/1, Eunsa, Pamplona 2002, 844; e tanti altri.

${ }^{54}$ I sacramenti costituiscono nel loro insieme un corpo armonico e strutturato, perciò si parla di organismo sacramentale, cfr. anche M. DEL Pozzo, La giustizia nel culto..., cit., 179-318. 
salvifici. I sacramenti sono diritti (beni dovuti) perché c'è una spettanza intrinseca alla lex gratiae derivante dalla mediazione del fattore umano. Al diritto di ricevere corrisponde appunto un dovere di amministrare secondo il dinamismo proprio dello $i u s^{55}$. Il sacramento è dunque necessariamente legato alla socialità ecclesiale e implica una specifica ministerialità (richiede un ministro), c'è quindi un vicolo e una relazione interpersonale. La radice dell'ordinamento sacramentale della Chiesa non a caso è stata individuata proprio nel principio nell'efficacia ex opere operato che rende oggettiva e concreta l'attribuzione ${ }^{56}$. L'indulgenza invece è solo il frutto dell'operato del fedele alle dovute condizioni e disposizioni. Nella fruizione dell'indulgenza manca appunto una prestazione obbligatoria e una relazione ad alios (il rapporto è direttamente con Dio). La giuridicità non è dunque intrinseca all'azione, legata alla natura dell'opera indulgenziata, ma deriva dalla previsione e assicurazione ecclesiale dell'effetto salvifico. L'indulgenza non è in definitiva un bene dovuto in giustizia coram hominibus.

$\mathrm{La}$ «giuridicità» dell'indulgenza è quindi più articolata e complessa. Fermo restando il fatto che ci muoviamo nell'ordine trascendente, non si tratta di un fenomeno congenito o costitutivo nella struttura della lex gratiae ma di un fattore acquisito o derivato attraverso l'autorità della Chiesa. Il divario non proviene tanto dal contrasto tra l'istituzione divina o l'istituzione ecclesiastica del bene, quanto dalla relazione di giustizia dedotta. L'indulgenza risponde alla giustizia legale e non ad una forma di giustizia lato sensu distributiva del patrimonio salvifico. Il profilo giuridico allora non recepisce un criterio di ripartizione fissato ex institutione divina, ma regola autonomamente l'attribuzione del beneficio e i presupposti del meccanismo remissivo. Il punto è che l'indulgenza si inserisce in una fattispecie a formazione progressiva che tra l'altro sovente implica l'economia sacramentale. Penitenza e Comunione evidentemente integrano le condizioni dell'indulgenza (almeno di quella plenaria), ma si tratta solo di componenti o elementi di un insieme composito, non costi-

55 Cfr. can. 843.

56 «L'efficacia dei sacramenti si fonda sui meriti di Cristo e perciò è oggettiva, ex opere operato, supposta la validità del segno e l'adeguata disposizione del soggetto. Dire ex opere operato equivale a dire ex meritis Christi [S. Th., III, q. 62, a. 5]. Meriti sovrabbondanti, preesistenti al sacramento, titolo perfecte de condigno, vale a dire in stretta e perfetta giustizia, che conferisce un'efficacia infallibile all'azione di Cristo» (J. HervadA, Le radici sacramentali..., cit., 641). Cfr. anche per un diverso approccio: A. M. RouCo VARELA - E. CORECCO, Sacramento e diritto: antinomia nella Chiesa? Riflessioni per una teologia del diritto canonico, Jaca Book, Milano 1971. 
tuiscono insomma il motivo del riconoscimento del surplus del condono o il fulcro attorno a cui ruota l'atto di clemenza. Il nucleo dell'istituto - ribadiamo - sfugge alla portata della giustizia. Se il fondamento dell'indulgenza risiede nella sovrabbondanza della Redenzione, il titolo sta nell'amministrazione ecclesiale del tesoro di grazie. La «pretesa» del perdono divino è legata allora a una misura autoritativa umana. Il diritto sta quindi nella facoltà di concessione e di regolazione e non certo nella logica e nella dinamica della condotta indulgenziata.

\section{I RILIEVI CIRCA IL FONDAMENTO E LA LOGICA AUTORITATIVA DEL BENE}

La giuridicità dunque riguarda solo la determinazione dei presupposti e la disciplina della fruizione dell'indulgenza, non concerne l'atteggiarsi e il contorno dell'attività indulgenziata (in quanto tale non interpersonale). La facoltà di normare o regolamentare appare però come una costante del governo ecclesiastico ${ }^{57}$. Il contenimento della portata giuridica non deve tuttavia portare a sottacere il carattere specifico e proprio del fenomeno. Le indulgenze sono diritto solo in quanto espressione positiva del potere di conformare autoritativamente la realtà? La risposta può essere affermativa e negativa allo stesso tempo. Affermativa in quanto il titolo e la misura del diritto sono indubbiamente legali. Negativa in quanto il bene radica nella funzione stessa della Chiesa, quale depositaria e garante del patrimonio salvifico. L'attribuzione della prerogativa costitutiva e regolativa (in cui si sostanzia la giuridicità delle indulgenze) è il riconoscimento o l'esplicazione della dimensione di giustizia di un dato preesistente (l'istituzionalità della Chiesa). Il necessario intervento della Chiesa (ope Ecclesiae) imprime così una decisa connotazione comunitaria e solidale al bene ${ }^{58}$.

Vale la pena approfondire perciò quella che abbiamo qualificato come «giuridicità radicale o originaria». La Chiesa è interprete qualificata del messaggio e dello strumentario divino di salvezza ${ }^{59}$. La stessa origine storica

${ }^{57}$ Nella visione dinamica di ciò che è giusto nella Chiesa emerge la configurazione del dovuto nel sistema giuridico-canonico, cfr. C. J. ERrázuriz M., Il diritto e la giustizia nella Chiesa. Per una teoria fondamentale del diritto canonico, Giuffrè Editore, Milano 2000, 213-223.

58 Il grembo della Madre è d'altronde il principale luogo d'incontro con la misericordia divina, cfr. J. REGO, Il grembo della misericordia. Sul linguaggio non-verbale alla base dell'Ordo Paenitentiae, Notitiae 51 (2015) 428-458.

59 Cfr. ad es. can. 841. La custodia ecclesiale del deposito di salvezza mira a rispettare e diffondere il mandato divino. 
dell'istituto è indicativa della presa di coscienza e della penetrazione del ruolo stimolante ed edificante della comunione dei santi ${ }^{60}$. La dottrina prima ancora che la disciplina delle indulgenze manifesta in pratica la maternità e accondiscendenza dei Pastori. La nascita dell'indulgenze non è stata dettata certo da una smania di potere o da una rivendicazione di dominio sulle coscienze, si è fatta strada nella percezione diffusa della gravosità del peccato e del rigore della penitenza quale forma di aiuto e incoraggiamento nella via del bene. L'intervento della Chiesa risulta allora integrativo e corroborativo della penitenza richiesta ${ }^{61}$. La dipendenza e l'appartenenza comunitaria (con il rilievo anche giuridico che assume la comunione ${ }^{62}$ ) marcano quindi il cammino di conversione e di remissione. L'indulgenza quindi è giuridica in quanto sostanzialmente e imprescindibilmente ecclesiale.

La dottrina normalmente sottolinea il legame tra indulgenza e giurisdizio$n e^{63}$. La radice dell'istituto si trova infatti nella potestà di giurisdizione $\mathrm{o}$, piuttosto, nella funzione di guida e di governo della comunità. Limitare però la considerazione del fenomeno all'aspetto potestativo è riduttivo e limitativo. Gli unici profili normativi indispensabili concernerebbero, come riportato ${ }^{64}$, la titolarità del potere e il rinvio alla legislazione del settore ${ }^{65}$. La convenienza epistemologica della stringatezza legale e del minimalismo codiciale non significa però disconoscere la chiave di volta del fenomeno indulgenziale. Lo ius, la valenza giuridica dell'attribuzione, come di consueto, conferisce oggettività, razionalità e concretezza alla spettanza. L'indulgenza non costituisce dunque una semplice emozione o sensazione liberatoria ma l'assicurazione dell'efficacia del percorso di riabilitazione e perdono fissato. Il discernimento

${ }^{60}$ Cfr. Catechismo della Chiesa Cattolica, nn. 1474-1477. J. Manzanares riferendosi al mistero della comunione dei santi aggiunge: «De esos bienes se participa a través de la indulgencia, no como camino fácil que sostituye la necesaria transformación del sujeto, sino como medio para favorecer esa transformación [nt. 54: Una de las preoccupaciones básicas de la reforma ha sido la de revalorizar la cooperación personal exigida al fiel]» (Penitencia, in J. MANZANARES MARIJUÁN A. Mostaza Rodríguez - J.L. Santos, Nuevo derecho parroquial, Editorial Católica, Madrid 1988, 293).

${ }^{61}$ L'ope Ecclesiae in pratica supporta l'efficacia ex opere operantis.

${ }^{62}$ Cfr. ad es. Nota explicativa praevia della Cost. dog. Lumen gentium, 16 novembre 1964, n. 2.

${ }^{63}$ Cfr. J.-M. GervaIs, La quarta edizione dell'Enchiridion..., cit., 179; C. CaCCIARI, Le indulgenze, 69-73.

${ }^{64}$ Cfr. supra nt. 43.

65 Osservava Gervais in riferimenti ai principi di carattere generale dei 6 canoni codiciali: «il vero e completo codice in materie di indulgenze è l'Enchiridion nella sua parte normativa» (La quarta edizione dell'Enchiridion..., cit., 177). 
ecclesiale inoltre manifesta la piena conformità dell'atto di liberalità con il piano personale e sociale di salvezza. Il potere costitutivo è sicuramente libero e discrezionale ma non è certo arbitrario e senza limiti ${ }^{66}$. La giuridicità del bene non si sostanzia poi in un valore ipotetico e astratto ma nell'accessibilità e incentivazione pratica del conseguimento del condono. La considerazione delle circostanze e del contesto esprime tutta l'umanità e il favore nei confront della realistica elargizione della promessa di grazia ${ }^{67}$. La remissio insomma non è tanto potestativamente confezionata quanto è configurata, interpretata e amministrata secondo giustizia (e carità).

In sede di redazione codiciale è stata affinata la determinazione del soggetto titolare del potere costitutivo. L'approfondimento ecclesiologico del Vaticano II ha portato infatti a riconoscere tale capacità in capo alla Suprema autorità e a determinare meglio le facoltà rimesse ai Vescovi diocesani ${ }^{68}$. L'autorità preposta può dunque concedere ex novo o abrogare indulgenze secondo il proprio giudizio prudenziale, rientrando nelle sue prerogative dispositive e distributive del patrimonio salvifico ${ }^{69}$. Tale dato evidenzia pure il carattere storico ed effettuale della funzione di guida (universale e particolare) della comunità. La tradizione del popolo di Dio d'altronde non è statica e immutabile ma dinamica e, per così dire, «lucrativa» ${ }^{70}$. Abbiamo già evidenziato che le indulgenze non sono solo autoritativamente previste ma opportunamente pianificate e ordinate. In linea con la visione partecipativa e comunitaria della realtà e missione cristiana emerse dall'assise conciliare, conviene anche sostituire alla concezione autoritaria e gerarchica, tipica dell'elaborazione della precedente codificazione, la logica del bene e del servi-

${ }^{66}$ Anche i temperamenti intervenuti a Trento, a fronte di abusi o irrispondenze comportamentali, indicano come la regolamentazione e disciplina fossero in linea con il bene delle anime.

${ }^{67}$ Basti pensare ai casi di commutazione o adeguazione nelle prescrizioni della concessione, cfr. ad es. ID nn. 11 e 18; Enchiridion Indulgentiarum, nn. 27-29.

68 «Oltre alla suprema autorità della Chiesa possono elargire indulgenze solamente quelli cui questa potestà viene riconosciuta dal diritto o è concessa dal Romano Pontefice» (can. $995 \$ 1 \mathrm{CIC}$ 83). La precisazione risale alla diseptatio de indulgetiis del marzo del 1972 (Communicationes 31 [1999] 328); il can. 912 CIC 17 riconosceva la spettanza ex institutione divina solo del Romano Pontefice («Praeter Romanum Pontificem, cui totius spiritualis Ecclesiae thesauri a Christo Domino commissa est dispensatio, ii tantum possunt potestate ordinaria indulgentias elargiri, quibus id expresse a iure concessum est»).

${ }^{69} \mathrm{La}$ decisione ovviamente, come già precisato, non è capricciosa e arbitraria ma risponde alla logica e proficuità della pratica penitenziale.

70 L'espressione si riferisce al mettere a frutto i talenti e le risorse ricevute. L'evoluzione o lo sviluppo disciplinare manifesta proprio l'incedere e il percorso che la Chiesa è chiamata a compiere nella realizzazione del piano divino. 
$z i o$. La costituzione delle indulgenze non rappresenta infatti una prerogativa o un privilegio della capitalità ma un fattore di crescita e incremento del Corpo. Fermo restando il carattere misterico e riservato del fenomeno, la facoltà istitutiva e regolativa non è una rivendicazione personale ma un'espressione carismatica del potere. Il patrimonio dei meriti e delle soddisfazioni di Cristo e dei santi integra non a caso i beni o l'essenza stessa della comunione. La gestione di tale ricchezza esprime quindi la portata diaconale e servente della potestà $^{71}$. Il ruolo direttivo dunque si sostanzia nella moderazione, promozione e custodia delle indulgenze ${ }^{72}$. La predisposizione o l'organizzazione non possono essere disgiunti dall'incentivo e dal controllo del costume penitenziale. Il compito promozionale dei Pastori non si esplica tanto nella creatività o originalità di previsione quanto nell'iniziativa e nella solerzia nel favorire la riconciliazione e la remissione dei fedeli ${ }^{73}$. Il desiderio della Chiesa d'altronde è che i suoi figli possano attingere sempre più copiosamente al tesoro della grazia.

Vale la pena ribadire allora che la giuridicità costitutiva richiamata implica che la dimensione di giustizia delle indulgenze è essenzialmente solidale e compartecipata nel popolo di Dio. Il riferimento istituzionale configura e qualifica il bene. Senza la vicarietà cristologica e la capitalità visibile mancherebbe in pratica la garanzia della disponibilità ed effettività della remissio. L'autorità conferisce quindi sicurezza e affidabilità al cammino di conversione e manifesta la base teologica e la valenza pastorale del profitto sperato.

\section{L'INCREMENTO «SOSTANZIALE» DELLA PRASSI INDULGENZIALE}

L'analisi compiuta ha evidenziato che il profilo giuridico non si limita alla configurazione e alla regolamentazione della remissione della pena (come per

$71 \ll$ Fra gli elementi che caratterizzano l'immagine vera e genuina della Chiesa, dobbiamo mettere in rilievo soprattutto questi: la dottrina, secondo la quale la Chiesa viene presentata come il popolo di Dio (cfr. Lumen gentium, 2) e l'autorità gerarchica viene proposta come servizio (cfr. ibid., 3), [...]» (GIOvanni PaOlo II, cost. ap. Sacrae disciplinae leges, qua Codex Iuris Canonici recognitus promulgatur, 25 gennaio 1983, AAS 75 [1983] II, XII).

72 L'elencazione proposta ha un riscontro nel ruolo relativo al culto sancito dal can. $835 \S 1$, che pare estensibile concettualmente in quest'ambito. Bisogna chiaramente distinguere l'incidenza e la portata del compito del Papa e dei singoli Vescovi.

73 L'atteggiamento e l'esempio personale di Papa Francesco è abbastanza eloquente e significativo (cfr. anche il contenuto di FranCESCO, Il nome di Dio è Misericordia. Una conversazione con Andrea Tornielli, Piemme, Città del Vaticano 2016). Cfr. inoltre le prescrizioni di GIOvanNI PaOLO II, m. p. Misericordia Dei, 7 aprile 2002, nn. 1-2, AAS 94 (2002) 455. 
ogni attività ecclesiale esterna) ma involge pure il presupposto e il contesto della fruizione del bene. Ciò non di meno la dimensione di giustizia non integra un connotato intrinseco alla realtà delle indulgenze. La giuridicità - come sopra illustrato - non è, per così dire, essenziale o costitutiva dell'azione prescritta (come per i sacramenti ${ }^{74}$ ) ma è iscritta (originariamente o radicalmente) nella logica della previsione, dipende in pratica dall'istituzionalità della Chiesa quale soggetto transpersonale e permanente ${ }^{75}$ e dai vincoli di solidarietà esistenti nella comunità cristiana. L'atteggiarsi della socialità d'altronde non è interno all'attività richiesta, deriva solo dal rapporto di comunione gerarchica. La relazione non riguarda dunque la ministerialità del dono di grazia (la mediazione umana dell'economia salvifica) ma l'efficacia e il contenuto della prescrizione normativa ${ }^{76}$. Il beneficio remissorio non è quindi oggetto proprio della virtù della giustizia, è espressione piuttosto della virtù di religione. Le indulgenze si inseriscono a pieno titolo nel novero dei beni ecclesiali e hanno quindi una portata giuridica ma non sono strettamente parlando beni giuridici o diritti ${ }^{77}$. Il problema non è chiaramente nel fondamento o nel titolo (ecclesiastico) del riconoscimento ma nella ratio e struttura stessa dell'attribuzione. Tale constatazione circoscrive epistemologicamente l'influenza dello ius, ma non sminuisce certo i compiti o il ruolo dell'autorità e dell'intero popolo di Dio.

Tornando al punto di partenza, l'inserimento nella disciplina codiciale risponde più ad una preoccupazione magisteriale e catechetica che ad una valutazione giuridico-concettuale ${ }^{78}$. La previsione rientra allora nell'impostazione teologica e dottrinale assunta dal Codificatore spesso evidenziata e talora

${ }^{74}$ I sacramenti sono anche beni propriamente giuridici, ma evidentemente trascendono ampliamente la dimensione di giustizia.

75 Cfr. J. Hervada, Diritto costituzionale canonico, Giuffrè Editore, Milano 1989, 158-164. Lo stesso Autore altrove precisa: «La Chiesa, essendo Ecclesia Spiritus, è veramente Ecclesia Iuris; e lo è non come due aspetti separabili ma in un'unità misterica nella quale la Ecclesia Iuris non è altro che una forma di manifestarsi nella storia umana dell'Ecclesia Spiritus» (Le radici sacramentali..., cit., 657).

${ }^{76}$ Nella dinamica indulgenziale (a prescindere dalla eventuale componente sacramentale) non si instaura una relazione ministro-fedele che implica una prestazione obbligatoria ma un'attività prettamente individuale e personale. Il rapporto Pastore-fedele resta precedente o esterno all'opera indulgenziata.

77 Si tratta di res sacrae iustae solo per la derivazione dalla titolarità e conformazione potestativa dell'elargizione.

${ }^{78}$ Nei lavori di redazione della codificazione latina invero mai si è supposto un abbandono completo del Capitolo de indulgentiis, dando per scontato che dovesse essere in qualche modo considerato e regolato. 
lamentata ${ }^{79}$. Limpianto normativo attuale ha optato per la scelta «intermedia» di un considerevole contenimento e alleggerimento del disposto senza però un mutamento di prospettiva troppo incisivo e radicale. La tradizione e la forma mentis diffusa sconsigliavano probabilmente cambiamenti più profondi e penetranti. L'ideale della completezza e praticità legislativa induceva peraltro a prevedere e regolare, almeno sommariamente, gli istituti principali. Sta di fatto che il can. 992 ( $m$ a in buona parte anche i cann. 993-994 ${ }^{80}$ ) hanno un contenuto prettamente definitorio e catechetico. Anche il can. 996 costituisce più un prontuario d'uso delle indulgenze che una precisa norma di condotta. Una sintesi della dottrina cattolica giova certamente alla chiarezza e alla divulgazione ma può ostacolare o confondere i piani di lettura della realtà ecclesiale ${ }^{81}$. Un riordino della giuridicità canonica e uno sviluppo ordinamentale ulteriore, frutto di una maturazione della sensibilità normativa in termini di essenzialità e rigore, dovrebbe forse portare ad uno sfrondamento del De indulgentiis che eviti accentuazioni giuridiciste o supplenze disciplinari ${ }^{82}$. Ł̀ indicativa tra l'altro la scelta operata dal $\mathrm{CCEO}^{83}$.

$\mathrm{Al}$ di là della consistenza delle prescrizioni, desta riserve anche la collocazione del Capitolo in appendice al sacramento della Penitenza. Il collegamento, come ricordato, ha un valore storico e pedagogico (evidenziando la necessità dello stato di grazia) ma non è troppo rispondente alla natura e all'inquadramento della concessione ${ }^{84}$. Le indulgenze si innestano nell'organismo sacra-

79 «La volontà di manifestare una grande sensibilità pastorale e di mostrare un volto benigno della disciplina ecclesiale forse hanno portato alla stesura di un Codice poco rigoroso sul piano tecnico (per esempio, con abbondanti esortazioni di impreciso valore giuridico), il che richiede nel giurista una maggiore attenzione al momento di proporre la soluzione giusta nel caso concreto» (E. BAuRA, Parte generale del diritto canonico. Diritto e sistema normativo, Edusc, Roma 2013, 241), anche L. Lorusso evidenzia nei sui commenti la natura prettamente teologica o teologico-giuridica di diversi canoni (Il culto divino nel Codex Canonum Ecclesiarum Orientalium. Commento ai singoli canoni, Ecumenica editrice, Bari 2008, 48-52, 66-68, 84-86, 94-95, ecc.).

${ }^{80}$ Cfr. anche J. A. Marques, Comentario cann. 992-994, in Comentario exegético..., cit., 844-847.

${ }^{81}$ In un'ottica giusrealista l'inquadramento teologico giova sicuramente alla percezione degli estremi dell'istituto ma può ostacolare la comprensione dei profili di doverosità intersoggettiva.

82 J. Hervada a proposito dei «vizi» della canonistica contemporanea, oltre alla deviazione del pastoralismo, ha denunciato spesso l'insidia del teologismo e dello pseudoteologismo (Pensieri di un canonista nell'ora presente, Marcianum Press, Venezia 2007, 9).

83 «CCEO: l'intero cap. [De indulgentiis] è omesso nel CCEO; tuttavia, anche gli orientali possono avvalersi delle indulgenze: cfr. Nuntia 10 (1980) 10 e art. $58 \$ 2$ PB» (l'annotazione è riportata in Codice di diritto canonico e leggi complementari commentato, ed. it. dir. J. I. ARRIETA, Coletti a San Pietro, Roma 2007, 662).

${ }^{84}$ Sembra trattarsi quasi di un supplemento perfettivo o integrativo della disciplina penitenziale. 
mentale ma non possono confondersi con l'istituzione divina del perdono sacramentale ${ }^{85}$. Una sistemazione più avveduta sembra rapportabile a Gli altri atti del culto divino, sempre che non si preferisca riferirlo semplicemente alla potestà configurativa dell'autorità nell'ambito della pietà o della carità ${ }^{86}$. L'esigenza a livello pastorale di evidenziare il presupposto e il legame logico della figura, specie in un'epoca di diffusa ignoranza e confusione religiosa, non esclude la ricerca di una maggior razionalità e rispondenza sistematica in sede legale.

Le considerazioni proposte non intendono minimamente inficiare o minare la pratica e la convenienza della remissione della pena, si propongono anzi di promuovere e incentivare lo spirito e il senso del perdono. L'origine e la diffusione delle indulgenze richiama prepotentemente il principio della salvezza: il primato della grazia e la chiamata alla contrizione-conversione. A prescindere dalla lodevole facilitazione del regime remissorio, l'intiepidimento del fervore ha oscurato in parte il nucleo stesso del cammino penitenziale ${ }^{87}$. La secolarizzazione e la perdita del senso del peccato hanno portano purtroppo a fraintendere il significato dell'invito e della prassi ecclesiale, svincolandolo dall'esigenza e serietà della riconciliazione ${ }^{88}$. Un merito, piuttosto recondito (perché difficilmente percepibile), della persistente esposizione della dottrina sulle indulgenze è la ricapitolazione degli elementi essenziali per la remissione. Benché la struttura o composita articolazione dell'attività lucrativa non è facilmente deducibile dal disposto, il testo legale ad ogni modo contie-

${ }^{85}$ Cfr. M. DEL Pozzo, La giustizia nel culto..., cit., 252-254. Ricorda E. Miragoli: «Per evitare il pericolo di confusione - le indulgenze, infatti, pur appartenendo all'economia sacramentale della Chiesa, non possono essere dette atti strettamente sacramentali - fu proposta, benché non attuata anche una collocazione diversa, cioè dopo la trattazione dei sette sacramenti (cfr. Communicationes 31 [1999] 322)» (Commento cc. 992-997, in Codice di diritto canonico commentato, a cura della Redazione di Quaderni di diritto ecclesiale, Áncora, Milano 2001, 805; per il riscontro cfr. supra nt. 39).

${ }^{86}$ Limitando la regolamentazione alla facoltà di concessione, la previsione potrebbe essere ricompresa nei canoni introduttivi del Libro IV.

87 L'indulgenza, come ricorda il Papa, più che un atto è un percorso o tragitto. La figura emblematica della Porta Santa ben lo evidenzia e ricorda: «Il pellegrinaggio è un segno peculiare nell'Anno Santo, perché è icona del cammino che ogni persona compie nella sua esistenza. [...] Anche per raggiungere la Porta Santa a Roma e in ogni altro luogo, ognuno dovrà compiere, secondo le proprie forze, un pellegrinaggio. Esso sarà un segno del fatto che anche la misericordia è una meta da raggiungere e che richiede impegno e sacrificio. Il pellegrinaggio, quindi, sia stimolo alla conversione [...]» (Misericordiae vultus, n. 14).

${ }^{88}$ Nell'immaginario collettivo spesso l'indulgenza giubilare è intesa come una sorta di amnistia o sconto di pena generalizzato per i fedeli. 
ne o riepiloga i fattori caratterizzanti della fattispecie, distinguendo opportunamente, al di là dell'esecuzione dell'opera prescritta, la capacità, l'intenzione, le disposizioni e le condizioni per lucrare un'indulgenza ${ }^{89}$. Capacità, intenzione e disposizioni sono d'altronde requisiti indispensabili per ogni ricezione di grazia. Nella catechesi una certa enfasi andrebbe posta proprio sull'attitudine e inclinazione del soggetto ${ }^{90}$. La meritevolezza del perdono non sta certo nei presunti meriti del fedele ma nell'accettazione «incondizionata» della misericordia divina. I requisiti sacramentali e devozionali integrano poi in maniera sostanziale la dinamica dell'affrancamento e dimostrano l'effettività e l'autenticità del desiderio di redenzione. Non bisogna dimenticare che la concessione autoritativa, pur con la liberalità e prodigalità del caso, apre quindi ad un percorso di contrizione e riabilitazione.

L'auspicabile incremento della prassi indulgenziale porta insomma ad evitare accuratamente deformazioni «magico-sacrali» nell'interpretazione del fenomeno e a riconoscere la coerenza e armonia dell'attribuzione ecclesiale. La spiritualità e interiorizzazione dell'azione risiede in buona parte nella maturazione delle disposizioni dovute (apte dispositus ${ }^{91}$ ) e nella promozione del perdono sacramentale ${ }^{92}$. La miglior definizione della portata giuridica delle indulgenze può contribuire perciò a scongiurare interpretazioni giuridiciste $o$ «meccanicistiche» dell'economia della grazia e a centrarsi sull'essenza della concessione ${ }^{93}$.

${ }^{89} \mathrm{Da}$ un punto di vista catechetico e pastorale sarebbe forse auspicabile che si fornisse una precisa scansione del procedimento remissorio: la coerenza interna del meccanismo spesso sfugge all'attenzione dei fedeli e ingenera mancanze o trascuratezze. Tale compito non appartiene evidentemente alla funzione di un codice ma ad un catechismo o vademecum (non ci risulta che un prontuario esplicativo e operativo sia presente nell'Enchiridion o nel Catechismo).

90 E ben noto il duro richiamo di S. Caterina da Genova: «Non ti fidare dicendo: "Mi confesserò, prenderò l'indulgenza plenaria e in quel momento sarò salvo perché purificato da tutti i miei peccati". Pensa che la confessione e la contrizione, che sono necessarie per l'indulgenza plenaria, sono così difficili d'avere che, se tu lo sapessi, tremeresti per la grande paura essendo più sicuro di non averla che di poterla avere» (CATERINA DA GENOva, Vita mirabile. Dialogo. Trattato sul purgatorio, a cura di F. Lovison, Città Nuova, Roma 2004, 258).

${ }^{91}$ Can. 992.

92 Commenta perciò G. Damizia: «Scompare definitivamente ogni carattere deterministico e quasi fisicistico delle indulgenze, in favore del suo vero e proprio aspetto spirituale e misterico. [...] Molto dipende dalle interne disposizioni del soggetto» (Commento c. 997, in P. V. PINTO [a cura di], Commento al Codice di Diritto Canonico, LEV, Città del Vaticano 2001, 599).

${ }^{93}$ Se in altre epoche l'inquadramento legale e le precisazioni normative incentivavano l'esecuzione del disposto (fino al rischio del rigorismo e dello scrupolo), oggi viceversa un'indebita accentuazione del profilo giuridico può sviare e allontanare dal nucleo principale della remissione (l'assoluzione sacramentale con la disposizione di una piena rettificazione). 


\section{Fonti}

Concilio di Clermont, c. 2, in J. D. Mansi (a cura di), Sacrorum Conciliorum nova et amplissima collectio, XX, Akademische Druck- und Verlaganstalt Graz, Graz 1960, col. 816.

Concilio di Trento, Decreto sulle indulgenze, 4 dicembre 1563, in Dz-Sch. 1835.

—, XXI sess., 16 luglio 1562, Decretum de reformatione, c. 9, in AA. Vv., Conciliorum Oecumenicorum Decreta, Edizioni Dehoniane, Bologna 1991, 731-732.

FrANCESCO, bolla Misericordiae vultus, 11 aprile 2015, AAS 107 (2015) 399-420.

Giovanni PaOlo II, m. p. Misericordia Dei, 7 aprile 2002, AAS 94 (2002) 452459.

-, cost. ap. Sacrae disciplinae leges, qua Codex Iuris Canonici recognitus promulgatur, 25 gennaio 1983, AAS 75 (1983) II, VII-XIV.

PaEnItentiaria Apostolica, Enchiridion indulgentiarum. Normae et concessiones, Typis Polyglottis Vaticanis-Libreria Editrice Vaticana, Città del Vaticano ${ }^{1} 1968,{ }^{4} 1999$.

PAOLO VI, cost. ap. Indulgentiarum doctrina, 1 gennaio 1967, AAS 59 (1967) 5-24.

Pontificio Consiglio Per i Testi Legislativi, Communicationes 13 (1981) 439-440; 15 (1983) 212-213; 31 (1999) 321-332; 32 (2000) 56, 120.

\section{Bibliografia}

Althaus, R., Komentar cc. 992-997, in Münsterischer Kommentar zum Codex iuris canonici, 4, Ed. K. Lüdicke, Ludgerus, Essen Januar 2008/Februar 2009.

BAura, E., Parte generale del diritto canonico. Diritto e sistema normativo, Edusc, Roma 2013.

CACCIARI, C., Le indulgenze, Edizioni Sabinae, Cantalupo in Sabina (RI) 2009.

-, Odierno sistema giuridico delle indulgenze e proposte di interpretazione, Pontificia Università San Tommaso d'Aquino, Roma 2007.

Calvo Espiga, A., Algunas orientaciones actuales de la teología de las indulgencias, Burgense 21 (1980) 417-449.

Cappello, F. M., Tractatus canonico-moralis de sacramentis, II, Marietti, TauriniRomae 1953.

Caterina da Genova - F. Lovison (a cura di), Vita mirabile. Dialogo. Trattato sul purgatorio, Città Nuova, Roma 2004. 
Dal Covolo, E., Per una storia dell'indulgenza. Nota in margine alla Bolla di indizione del Grande Giubileo, Salesianum 61 (1999) 819-829.

Damizia, G., Commento c. 997, in P. V. PinTo (a cura di), Commento al Codice di Diritto Canonico, LEV, Città del Vaticano 2001, 599.

Del Pozzo, M., La giustizia nel culto. Profili giuridici della liturgia della Chiesa, Edusc, Roma 2013.

—, La doverosità liturgica, morale e giuridica del culto ecclesiale, Ius Ecclesiae 21 (2009) 549-568.

—, La dimensione giuridica della liturgia. Saggi su ciò che è giusto nella celebrazione del mistero pasquale, Giuffrè Editore, Milano 2008.

De Paolis, V., Il sacramento della Penitenza, in A. Longhitano - A. Montan - J. Manzanares - V. DE PaOlis - G. Ghirlanda, I sacramenti della Chiesa, Edizioni Dehoniane, Bologna 1989, 233-237.

De Pascal, G., Les indulgences. Doctrine et histoire, Bloud et C., Paris 1908.

ERráZURIZ M., C. J., Lintrinseca doverosità liturgica e giuridica del culto ecclesia$l e$, in E. BAURA - M. DEL Pozzo (a cura di), Diritto e norma nella liturgia, Giuffrè Editore, Milano 2016, 29-58.

—, Il diritto e la giustizia nella Chiesa. Per una teoria fondamentale del diritto canonico, Giuffrè Editore, Milano 2000.

Francesco, Il nome di Dio è Misericordia. Una conversazione con Andrea Tornielli, Piemme, Città del Vaticano 2016.

Frank, E., I Sacramenti dell'Iniziazione, della Penitenza e dell'Unzione degli infermi. Commento ai Canoni 834-1007 del Codice di Diritto Canonico, Urbaniana University Press, Città del Vaticano 2012.

Galtier, P., De paenitentia. Tractatus dogmatico-historicus, apud aedes Universitatis Gregorianae, Romae 1956.

—, Les indulgens: origine et nature, à propos d'un ouvrage récent, Gregorianum 31 (1950) 258-274.

GERVAIS, J.-M., La quarta edizione dell'Enchiridion Indulgentiarum, Ius Ecclesiae 12 (2000) 173-187.

GiacobBI, A., Storia della disciplina penitenziale antica, Roma 1976.

GoÑ GaZTAmBiDe, J., Las primeras indulgencias de difuntos, Antologica Annua 2 (1954) 378-391.

Hervada, J., Pensieri di un canonista nell'ora presente, Marcianum Press, Venezia 2007.

—, Le radici sacramentali del diritto canonico, Ius Ecclesiae 17 (2005) 629-658. 
—, Diritto costituzionale canonico, Giuffrè Editore, Milano 1989.

Jungmann, J. A., Die lateinischen Bussriten in ibrer geschichtlichen Entwicklung, F. Rauch, Innsbruck 1932.

LÉPICIER, A. H. M., Le indulgenze. Loro origine, natura, e svolgimento, Direzione della Biblioteca del clero, Siena 1897.

LORÍA, R., La Penitenza nei secoli. Excursus storico sullistituto penitenziale nella Chiesa, in L. LigIER ET AL. (a cura di), La penitenza. Dottrina, storia, catechesi e pastorale, Elledici, Torino 1967, 177-224.

Lorusso, L., Il culto divino nel Codex Canonum Ecclesiarum Orientalium. Commento ai singoli canoni, Ecumenica editrice, Bari 2008.

Manzanares, J., Penitencia, in J. Manzanares Marijuán - A. Mostaza RodríGUEZ - J. L. SANTOS (eds.), Nuevo derecho parroquial, Editorial Católica, Madrid 1988.

Marques, J. A., Comentario c. 992, in Á. MarzoA - J. Miras - R. RodríguezOCAÑa (eds.), Comentario exegético al Código de Derecho Canónico, III/1, Eunsa, Pamplona 2002, 844-845.

MigliavaccA, A., Le indulgenze, Quaderni di diritto ecclesiale 11 (1998) 159-177.

Miragoli, E., Commento cc. 992-997, in RedaZIone Di Quaderni Di DiRITTO ECCLESIALE (a cura della), Codice di diritto canonico commentato, Áncora, Milano 2001, 805-809.

Paulus, N., Geschichte des Ablasses im Mittelalter, I-III, Paderborn 1922-1923, rist. WBG, Darmstadt 2000.

Peters, E. N., Incrementa in progressu 1983 Codicis iuris canonici, Wilson \& Lafleur, Montréal 2005.

Pighin, B. F., Diritto sacramentale, Marcianum Press, Venezia 2006.

Poschmann, B., Der Ablass im Licht der Bussgeschicbte, Bonn 1948 (Theophaneia 4).

Poschmann, B. - Courtney, F., Penance and the anointing of the sick, HerderBurns and Oates, Freiburg im Breisgau-London 1964.

Regatillo, E. F., Las indulgencias, Sal Terrae, Santander 1947.

Rego, J., Il grembo della misericordia. Sul linguaggio non-verbale alla base dell'Ordo Paenitentiae, Notitiae 51 (2015) 428-458.

Rouco Varela, A. M. - Corecco, E., Sacramento e diritto: antinomia nella Chiesa? Riflessioni per una teologia del diritto canonico, Jaca Book, Milano 1971.

RUIZ MAINARDI, R. D., Indulgencias, in Diccionario general de derecho canónico, en J. OTADUY - A. Viana - J. SEDANo (eds.), Diccionario general de derecho 
canónico, IV, Thomson Reuters Aranzadi, Cizur Menor (Navarra) 2012, 551-554.

-, La disciplina de las indulgencias en el siglo XX, Pontificia Universitas Sanctae Crucis, Romae 2000.

TauninkL, W., La discussione teologica sulle indulgenze dal Concilio Vaticano II fino ad oggi, Galaxia Gutenberg, Târgu Lapus 2005.

Urru, A., La funzione di santificare della Chiesa [I Sacramenti], Pontificia Università di San Tommaso d'Aquino in Roma, Roma 1987.

Vogel, C., Il peccatore e la penitenza nel medioevo, Elledici, Torino 1988.

—, Il peccatore e la penitenza nella Chiesa antica, Elledici, Torino 1967.

Woestman, W. H., Sacraments. Initiation, Penance, Anointing of the Sick. Commentary on Canons 840-1007, Saint Paul University, Ottawa 2004. 
COMENTARIOS Y NOTAS 
